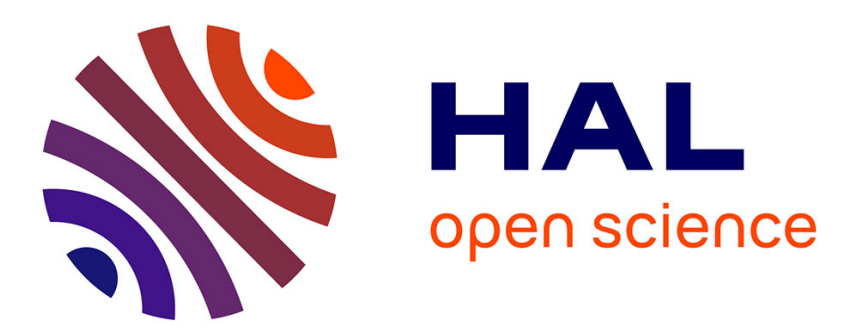

\title{
Repetitive experiments of one or two-pulse sequences in NQR of spins I=3/2: Liouville space, steady-state, Ernst angle and optimum signal \\ Christophe Odin
}

\section{- To cite this version: \\ Christophe Odin. Repetitive experiments of one or two-pulse sequences in NQR of spins I=3/2: Liou- ville space, steady-state, Ernst angle and optimum signal. Solid State Nuclear Magnetic Resonance, 2017, 85, pp.25-33. 10.1016/j.ssnmr.2017.04.004 . hal-01580454}

HAL Id: hal-01580454

https://hal-univ-rennes1.archives-ouvertes.fr/hal-01580454

Submitted on 16 May 2018

HAL is a multi-disciplinary open access archive for the deposit and dissemination of scientific research documents, whether they are published or not. The documents may come from teaching and research institutions in France or abroad, or from public or private research centers.
L'archive ouverte pluridisciplinaire $\mathbf{H A L}$, est destinée au dépôt et à la diffusion de documents scientifiques de niveau recherche, publiés ou non, émanant des établissements d'enseignement et de recherche français ou étrangers, des laboratoires publics ou privés. 


\title{
Repetitive experiments of one or two-pulse sequences in NQR of spins $I=3 / 2$ : Liouville space, steady-state, Ernst angle and optimum signal.
}

\author{
Christophe ODIN \\ ${ }^{1}$ Institut of Physics of Rennes IPR/UMR CNRS 6251, University of Rennes I, Campus \\ de Beaulieu, Bat 11A, 35042 Rennes Cedex, FRANCE
}

\section{Abstract}

In NMR, the repetition of pulse sequences with a recycle time that does not allow the spin system to completely relax back to equilibrium is a well known and often used method to increase the signal to noise ratio at given total measuring time. For isolated spins $I=1 / 2$, the steady-state of a train of strictly identical pulse sequences separated by free evolution periods of same duration is described by the well known Ernst-Anderson model, and the optimum pulse angle is given by the Ernst angle. We showed recently that equivalent formula, but with super-operators in the Liouville space, can be obtained for general spins I. In this article, this formalism is generalized to pure NQR of spins $I=3 / 2$, and applied to calculate the signal resulting from single and solid-echo sequences, in the limit when the recycle time $T>5 T_{2 q}$, where $T_{2 q}$ is the transverse (coherence) quadrupolar relaxation time. In particular, we show that powder samples have a behaviour that is very close to NMR of spins $I=1 / 2$. For instance, the generalized Ernst

Email address: christophe.odin@univ-rennes1.fr (Christophe ODIN) 
angle $\beta_{M}$ that maximizes the signal amplitude for a single pulse train is well described by the simple formula $\cos \left(1.52 \beta_{M}\right) \approx \exp \left(-T / T_{1 q}\right)$, whatever the quadrupolar asymmetry parameter $\eta, T_{1 q}$ being the longitudinal (population) quadrupolar relaxation time. Moreover, a simplified NMR-like formula that describes the overall behaviour of nutation curves is proposed, and it is shown that the signal to noise ratio (SNR) at given experimental time is exactly the same as in NMR of spins $\mathrm{I}=1 / 2$ as a function of recycle time, when properly normalized. Some theoretical predictions for the single pulse and solid-echo sequence were compared to experiments, and validated, by performing ${ }^{35} \mathrm{Cl}$ pure NQR experiment on chloranil $\left(\mathrm{C}_{6} \mathrm{Cl}_{4} \mathrm{O}_{2}\right.$ tetrachloro-1,4-benzoquinone) powder.

Keywords: Nuclear Quadrupole Resonance NQR, Liouville Space, Progressive Saturation, steady-state, Spins I=3/2, ${ }^{35} \mathrm{Cl}$ NQR, Ernst angle, chloranil

\section{Introduction}

Nuclear Quadrupole Resonance is a powerful resonance method that probes directly the splitting of the energy levels of the spins $I \geq 1$ by the quadrupolar interaction[1],[2]. Since its discovery in the 1950s [3], [4], [5], [6], [7], [8] , it is widely used in condensed-matter physics, for instance for the study of phase transitions or polymorphism, and has also biophysical or pharmaceutical applications [9],[10], [11], [12],[13], [14].

The experimental NQR set-up is identical to NMR, but no strong static external magnetic field is needed. NQR has advantages and drawbacks compared to NMR. In NMR, the Zeeman interaction is the dominant interaction, 
and all other interactions (dipolar, chemical shift, quadrupolar, etc...) should be treated as a perturbation to first or second order. Thus, the value of the resonance frequency is mainly given by the Zeeman interaction, and represents typically a few ppm of the main Larmor frequency. The frequency shift depends on the relative orientation of the Principle Axis System (PAS) of the interaction tensor with respect to the main static magnetic field, and powder samples lead to broad powder spectra with typical shapes that include steps and divergences. The excitation by the radio-frequency (RF) field may be orientation dependent if the RF power is not strong compared to the truncated dipolar, CSA or quadrupolar interactions.

In contrast to NMR, the quadrupolar interaction is the main interaction in NQR : dipolar and Zeeman interactions are small compared to the quadrupolar interaction. As the interaction is directly probed by the RF field, the splitting of the energy levels that depends on the mean quadrupolar interaction are strong function of temperature, pressure, or any small perturbation that affects the Electric Field Gradient (EFG) at the nuclei (bond lengths, strains, local defects, charge transfer etc...). This sensitivity that can be used to detect very small structural or electronic changes, is one of the main advantages of NQR. But it is also its main drawback because the NQR resonance frequency is not known a priori, and may be long to be found. In NQR, both excitation and detection depend on the relative orientation of the coil with respect to the PAS of the EFG. In particular, when a powder sample with a single quadrupolar site in NMR would give a broad spectrum because all PAS orientations with respect to the external static magnetic field have a different frequency, in NQR only one line is observed, 
because the energy splitting is not a function of orientation

Experimentalist have always been concerned with the problem to determine the optimum conditions to obtain an improvement in signal to noise ratio in NMR and NQR experiments, because of their low sensitivity. The co-addition of signals from successive experiments imposes that the recycle time should be at least five times longer than the longest relaxation time of the system, to ensure that the initial state is at thermodynamical equilibrium. In NMR, the question of whether experiments could be performed with shorter recycle times has been addressed as early as 1958 [15], in particular the importance of the steady-state that results of the competition between the pulses of the sequence that create and mix-up populations and coherences, and the free evolution periods where populations and coherences relax back to their equilibrium values.

The theory of progressive saturation experiments developed by Ernst and Anderson [16] for isolated spins $I=1 / 2$ calculated the effects on the NMR signal of the repetition of identical single pulses separated by a recycle time not long enough to allow the system to relax completely to its thermal equilibrium state. The theory considered the contribution of both longitudinal $T_{1}$ and transversal $T_{2}$ relaxation times $[16,17,18,19]$. Simple results were obtained when the coherences have been irreversibly cancelled by some means (for instance with a strong field gradient pulse applied between two RF pulses), it was shown that the longitudinal magnetization attains a well defined steadystate during the recycle time.

For a hard pulse of angle $\beta$ applied at resonance, the well known and 
celebrated Ernst-Anderson formula for $\mathrm{I}=1 / 2$ are

$$
\begin{gathered}
M_{s s t}=\frac{1-e^{-T / T_{1}}}{1-\cos (\beta) e^{-T / T_{1}}} M_{e q} \\
S_{s s t}^{N M R}(\beta)=\frac{1-e^{-T / T_{1}}}{1-\cos (\beta) e^{-T / T_{1}}} \sin (\beta) M_{e q}
\end{gathered}
$$

where $M_{s s t}$ and $S_{s s t}^{N M R}$ are the steady-state magnetization and signal respectively, $\mathrm{T}$ is the recycle time. The signal at given recycle time $T$ presents a maximum as a function of pulse length for the Ernst angle

$$
\cos (\beta)=e^{-T / T_{1}}
$$

The aim of this article is to consider the case of NQR of spins $I=3 / 2$. To the best of our knowledge, the steady-state of fast repetitive experiments in NQR was only considered for $I=1$ spins [20] for single pulse sequence and complete decay of the coherences during the recycle time, and compared to ${ }^{14} N$ NQR experiments. In this article, we consider NQR of spins I=3/2. This is quite general, because from a periodic table of quadrupolar nuclei [21], about $40 \%$ of isotopes have a spin $I=3 / 2$. In NQR, most of the $I=3 / 2$ studied nuclei are ${ }^{35} \mathrm{Cl}$ (75.5\% of natural isotopic abundance NIA), ${ }^{63} \mathrm{Cu}$ $(\mathrm{NIA}=69.1 \%),{ }^{81} \mathrm{Br}(\mathrm{NIA}=49.5 \%)$.

Recently, we calculated in the Liouville space the steady-state density vector of a system subjected to a train of single or two-pulse sequences $([22])$, with periodic repetition at the recycle time rate. The formula were obtained as a function of the pulse and free-evolution super-operators with a few hypothesis, and can also be applied to NQR. By combining these formal solutions to a formalism that we developed for NQR of spins $3 / 2$ [23], [24], it 
is shown in this article that explicit formula for the signal, in the hypothesis that the coherences have decayed during the recycle time, can be obtained. An analogy with the Ernst-Anderson model developed for NMR of spins $\mathrm{I}=1 / 2$ and $\mathrm{NQR}$ of spins $\mathrm{I}=3 / 2$ is also drawn.

This article is divided as follows. Section 2 presents a summary of spin $\mathrm{I}=3 / 2$ NQR formalism and Liouville space calculation of steady-state equilibrium. Both formalisms are then applied to NQR of spins $I=3 / 2$. In particular, free-evolution and pulse super-operators are given, and the general solutions in the Liouville space are presented for one or two-pulse repetitive experiments. In section 3, explicit formula for on or out of resonance pulses applied to powder samples are obtained and analysed. Theoretical results and simulations are compared to experimental data obtained from ${ }^{35} \mathrm{Cl}$ NQR of chloranil $\left(\mathrm{C}_{6} \mathrm{O}_{2} \mathrm{Cl}_{4}\right.$ 2,3,5,6-Tetrachloro-p-Benzoquinone) at ambient temperature.

\section{Theory of repetitive experiments in NQR of spins $I=3 / 2$}

The continuous excitation of a system by periodic pulse sequences, with a period given by the recycle time $T$, will produce a steady-state that is independent of the initial conditions. However, the resulting steady-state depends on both the pulse sequence and how the recycle time compares to the population and coherence relaxation times. Recently, we calculated a general solution for a one and two-pulse sequence in the Liouville space ([22]), from rather general hypothesis. Moreover, we developed a simplified formalism of NQR of spins $\mathrm{I}=3 / 2([23],[24])$, that can be directly used in

the Liouville space. The aim of this section is to present a summary of the 
steady-state solutions obtained in the Liouville space, and to apply them to NQR of spins $I=3 / 2$.

\subsection{Steady-state in the Liouville space}

In the Liouville space, the density vector $|\rho\rangle$ obeys equation

$$
\frac{\partial|\rho\rangle}{\partial t}=\mathbb{L}\left(|\rho(t)\rangle-\left|\rho_{e q}\right\rangle\right)
$$

with $\mathbb{L}=\left(-i \mathcal{H}^{l}+\mathbb{R}\right)$ the Liouvillian. $\mathcal{H}^{l}$ is the Liouville super-operator associated to the interaction hamiltonian, and $\mathbb{R}$ is the relaxation superoperator. $\left|\rho_{e q}\right\rangle$ is the equilibrium density vector.

The formal solution of Eq.[4] for free-evolution is

$$
|\rho(t)\rangle=\mathbb{E}_{t}|\rho(0)\rangle+\left(\mathbb{I}-\mathbb{E}_{t}\right)\left|\rho_{e q}\right\rangle \text { with } \mathbb{E}_{t}=e^{\mathbb{L} t}
$$

where $\mathbb{I}$ is the identity operator. The free evolution propagator operator $\mathbb{E}_{t}$ is non unitary because relaxation is an irreversible process. The two superoperators $\mathcal{H}^{l}$ and $\mathbb{R}$ do not commute in general, so that the free evolution propagator is not the product of two exponential super-operators.

Given a pulse sequence that consists of pulses of rectangular shape, $R F$ power and phase, and free evolution periods of fixed duration, a repetitive experiment is the repetition of this pulse sequence followed by a free evolution period of duration $\mathrm{T}$. The repetition is periodic with a period of length close to $\mathrm{T}$.

Such experiments are illustrated in Fig.1(left), for a single pulse or solidecho repetitive experiments. The pulses mix populations and coherences, while the free evolution period mix coherences of same order, and drives 

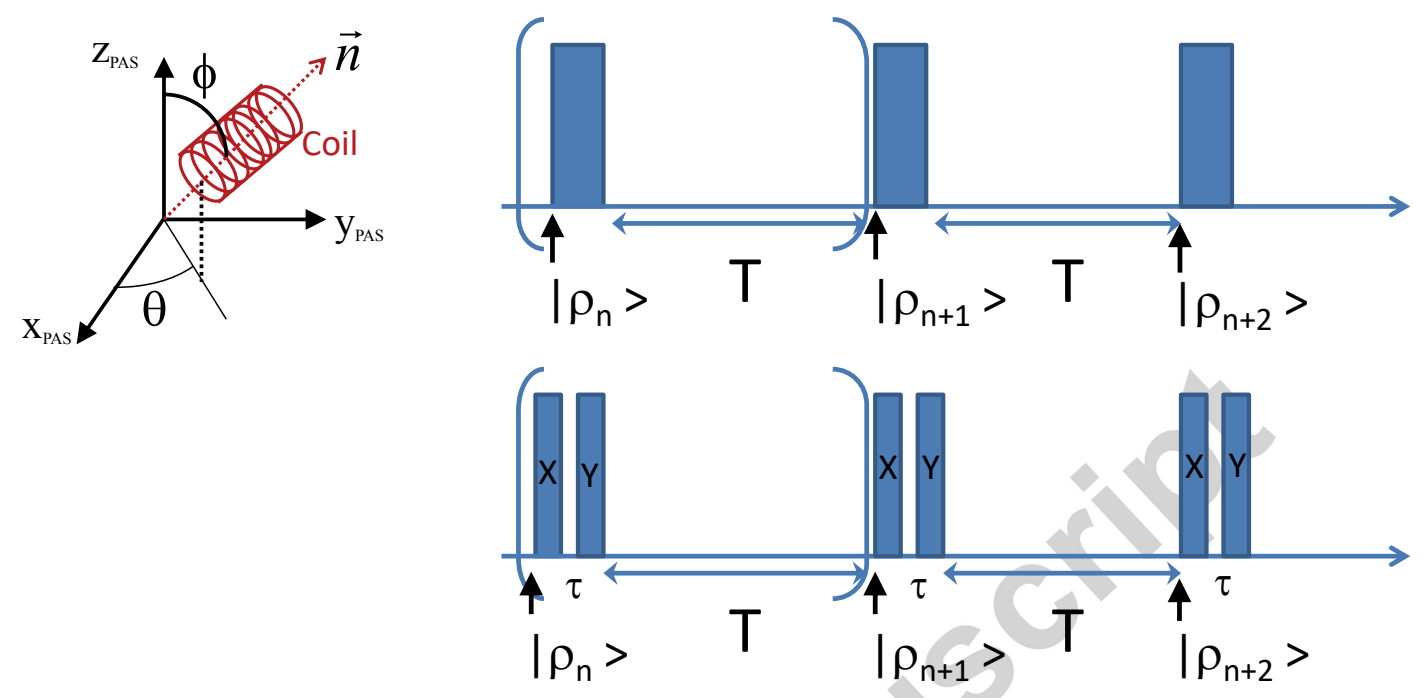

Figure 1: (Left) NQR set-up with the orientation of the coil with respect to the PAS of the EFG tensor. (Right) Repetitive experiments sequences.(Top) Single pulse sequence ;(Bottom) Two-pulse sequence. After a large number of repetitions, the steady-state is independent of the initial conditions, whatever the recycle time. If the recycle time is long enough, typically larger than five times the largest relaxation time, then at each iteration, the density vector before the sequence relaxed to the equilibrium density vector $\left|\rho_{n}\right\rangle=\left|\rho_{e q}\right\rangle$.

them back to equilibrium. At equilibrium, the coherences have decayed to zero, and the populations are fixed by thermodynamical equilibrium. When the recycle time is much longer than the largest relaxation time, the density matrix before each sequence is the equilibrium value and each sequence starts with the same initial density vector. On the other hand, when the recycle time has not allowed the system to relax back to equilibrium during the recycle time, a competition arises between the pulses and the free evolution periods that leads to a steady-state due to a dynamical equilibrium. 
The steady-state solutions were calculated in [22]. For a one pulse sequence, we obtained

$$
\left|\rho_{s s t}\right\rangle=(\mathbb{I}-\mathbb{E} \mathbb{P})^{-1}(\mathbb{I}-\mathbb{E})\left|\rho_{e q}\right\rangle
$$

where the free evolution (including relaxation) of duration $\mathrm{T}$ is represented by the super-operator $\mathbb{E}=\mathbb{E}_{T}$, and $\mathbb{P}$ is the pulse super-operator. Equation.6 is the super-operator generalization for a general spin $I \geq 1 / 2$ of the ErnstAnderson equation obtained for NMR of an isolated spin $I=1 / 2$. The steadystate is independent of the initial conditions.

For a two pulse sequence $\left(P_{1}\right)-\tau_{2}-\left(P_{2}\right)-T-$,

$$
\left.\left|\rho_{s s t}\right\rangle=\left(\mathbb{I}-\mathbb{E P}_{2} \mathbb{E}_{\tau_{2}} \mathbb{P}_{1}\right)\right)^{-1}\left(\mathbb{I}-\mathbb{E}+\mathbb{E P}_{2}\left(\mathbb{I}-\mathbb{E}_{\tau_{2}}\right)\right)\left|\rho_{\text {eq }}\right\rangle
$$

\section{2. $N Q R$ of spins 3/2}

In NQR, the quadrupolar interaction is considered as being much larger than other interactions : dipolar interactions, Zeeman interaction if a small static magnetic field is present, and the Radio-Frequency (RF) field Hamiltonian when a pulse is applied. Exact solutions for NQR of spins $\mathrm{I}=3 / 2$ were obtained by Pratt et al. [25]. In this section, we summarize the principal equations of the formalism that we developed in [23] for the special case of spins $\mathrm{I}=3 / 2$. The Zeeman and dipolar interactions are neglected, to treat only the case of a spin subjected to a strong quadrupolar interaction and a RF field in the coil direction.

The principal axis system PAS of the Electric Field Gradient (EFG) is oriented with its $\mathrm{z}$ axis along the eigenvector corresponding to the largest 
eigenvalue of the EFG in absolute value. The spin operators are defined in this PAS. In the PAS, the quadrupolar hamiltonian is then

$$
H_{q}=\frac{e M_{Q} V_{z z}}{12}\left\{3 I_{z}^{2}-I(I+1)+\frac{\eta}{2}\left(I_{+}^{2}+I_{-}^{2}\right)\right\}
$$

where $M_{Q}$ is the quadrupole moment, $\eta=\frac{V_{x x}-V_{y y}}{V_{z z}}$ the EFG asymmetry parameter $0 \leq \eta \leq 1$ with the convention $\left|V_{x x}\right| \leq\left|V_{y y}\right| \leq\left|V_{z z}\right|$, and $V_{z z}=e q$ the EFG at the nucleus under study.

During a pulse of rectangular shape, at irradiation frequency $\omega$, and amplitude $\propto \omega_{R F}$, the Hamiltonian is written has

$$
H_{T}=H_{q}+H_{R F}=H_{q}+2 \omega_{R F} \cos (\omega t+\varphi) \mathbf{I} . \mathbf{n}
$$

with $\mathbf{n}=(\sin (\theta) \cos (\phi), \sin (\theta) \sin (\phi), \cos (\theta))$ the unit vector giving the coil axis direction in the PAS (Fig.1(left)).

When $I=\frac{3}{2}$, the quadrupolar hamiltonian has two twofold eigenvalues of opposite sign $\pm \frac{e^{2} q M_{Q}}{2} \sqrt{1+\frac{\eta^{2}}{3}}$, giving the corresponding NQR angular resonance frequency :

$$
\omega_{q}=\frac{e^{2} q M_{Q}}{2} \sqrt{1+\frac{\eta^{2}}{3}}
$$

From the Cayley-Hamilton theorem, we deduce that the quadrupolar Hamiltonian is proportional to an operator $Q$ that is its own inverse :

$$
\begin{gathered}
H_{q}=\frac{\omega_{q}}{2} Q \\
Q^{2}=I_{4}
\end{gathered}
$$

with $I_{4}$ the $4 \mathrm{x} 4$ identity matrix. 
From this formal property, we derived a formalism to calculate the evolution of the operators during a free evolution period, and during a rectangular pulse. Some interesting analogies with the case of spins $\mathrm{I}=1 / 2$ can be derived [23].

The equilibrium density operator is :

$$
\rho_{e q}=\frac{e^{-\frac{H_{q}}{k T}}}{\operatorname{Tr}\left(e^{-\frac{H_{q}}{k T}}\right)}=\frac{I_{4}}{4}-\tanh \left(\frac{\omega_{q}}{2 k T}\right) \frac{Q}{4}
$$

In the interaction representation, the Hamiltonian during a pulse becomes

$$
H=\Delta \omega Q_{z}^{\lambda}+\lambda^{\prime}(\theta, \phi) \omega_{R F}\left[\cos (\xi) Q_{x}^{\lambda}+\sin (\xi) Q_{y}^{\lambda}\right]
$$

where $\lambda^{\prime}(\theta, \phi)$ is a directional parameter (see below). The normalized operators $Q_{x}^{\lambda}, Q_{y}^{\lambda}, Q_{z}^{\lambda}\left(\operatorname{Tr}\left(\left(Q_{\alpha}^{\lambda}\right)^{2}\right)=1\right)$ fulfil the usual commutation relations as Cartesian spin operators

$$
\left[Q_{x}^{\lambda}, Q_{y}^{\lambda}\right]=i Q_{z}^{\lambda} \text { and cyclic permutations }
$$

$\left\{Q_{x}^{\lambda}, Q_{y}^{\lambda}, Q_{z}^{\lambda}\right\}$ is an orthonormal basis, $\operatorname{Tr}\left(Q_{\alpha}^{\lambda} Q_{\beta}^{\lambda}\right)=\delta_{\alpha \beta}$. Note that $Q_{z}^{\lambda}=$ $Q / 2$ does not depend on the directional parameter, as expected on physical grounds. In this basis, and forgetting all irrelevant factors and the identity matrix that do not contribute to the signal, $\rho_{e q} \sim Q_{z}^{\lambda} \sim Q_{z}$.

Angle $\xi=\pi / 2+\varphi, \Delta \omega=\omega-\omega_{q}$ is the difference between the angular irradiation frequency and the quadrupolar frequency, and the directional parameter $\lambda^{\prime}(\theta, \phi)$ is defined by

$$
\lambda^{\prime}(\theta, \phi)=\frac{1}{\sqrt{3+\eta^{2}}} \sqrt{4 \eta^{2} \cos ^{2}(\theta)+\sin ^{2}(\theta)\left[9+\eta^{2}+6 \eta \cos (2 \phi)\right]}
$$


The minimum and maximum value of the directional parameter are :

$$
\begin{aligned}
\lambda_{\text {min }}^{\prime} & =\frac{\eta}{\sqrt{3+\eta^{2}}} \text { coil along zPAS axis } \\
\lambda_{\max }^{\prime} & =\frac{3+\eta}{\sqrt{3+\eta^{2}}} \text { coil along xPAS axis }
\end{aligned}
$$

It is interesting to note that the directional parameter $\lambda^{\prime}$ averaged over all orientations is a slightly increasing function of $\eta$, that varies from $\left\langle\lambda^{\prime}\right\rangle_{(\eta=0)} \approx 1.3598$ to $\left\langle\lambda^{\prime}\right\rangle_{(\eta=1)} \approx 1.3804$, while its standard deviation decreases as $\sqrt{<\left(\Delta \lambda^{\prime}\right)_{(\eta=0)}^{2}>} \approx 0.3877$ to $\sqrt{<\left(\Delta \lambda^{\prime}\right)_{(\eta=1)}^{2}>} \approx 0.3087$.

For a given direction of the coil with respect to the PAS of the EFG, the signal is

$$
S\left(t, \lambda^{\prime}\right)=\lambda^{\prime} \operatorname{Tr}\left(Q_{+}^{\lambda} \rho(t)\right)
$$

where $Q_{+}^{\lambda}=Q_{x}^{\lambda}+i Q_{y}^{\lambda}$. Equations.12 and 14 show that in NQR, although the resonance frequency is independent of the relative orientation of the coil with respect to the EFG PAS, the intensity is a strong function of the direction. The intensity depends on parameter $\lambda^{\prime}(\theta, \phi)$ from both the excitation (through an effective radiofrequency power $\lambda^{\prime} \omega_{R F}$ ) and the detection with a factor $\lambda^{\prime}$.

Note the analogy with NMR of spins $I=1 / 2$, if we assume an effective radiofrequency power $\lambda^{\prime} \omega_{R F}$, and a detection factor of $\lambda^{\prime}$. In pure NMR, we would have $\lambda^{\prime}=1$.

All results given above are valid for a single orientation of the coil with respect to the PAS, that is for a single crystal. When the sample is an isotropic powder, the signal is the average other all orientations $\theta$ et $\phi$. In 
fact, it is easier, either analytically or numerically, to perform the average over $\lambda^{\prime}$ using the probability density $g\left(\lambda^{\prime}\right)$ :

$$
S(t)=<S\left(t, \lambda^{\prime}\right)>=\int_{\lambda_{\text {min }}^{\prime}}^{\lambda_{\text {max }}^{\prime}} S\left(t, \lambda^{\prime}\right) g\left(\lambda^{\prime}\right) d \lambda^{\prime}
$$

An analytical expression of $g\left(\lambda^{\prime}\right)$ as a function of the complete elliptic integral can be found in [23]. From the numerical point of view, it is much easier to perform the average in Eq.15 using a Monte-Carlo integration method, than to use the analytic expression of $g\left(\lambda^{\prime}\right)$, because it contains a divergence, and the numerical evaluation of complete elliptic integrals is quite computer demanding.

\subsection{Steady-state in repetitive experiments of NQR of spins 3/2}

Using the results of the previous subsections, we see that for a given directional parameter $\lambda^{\prime}$, a Liouville space $L_{\lambda}$ can be defined, of dimension 3. Density vectors can be defined as :

$$
\left|\rho^{\lambda}(t)\right\rangle=\left(\begin{array}{c}
\rho_{z}^{\lambda}(t) \\
\rho_{x}^{\lambda}(t) \\
\rho_{y}^{\lambda}(t)
\end{array}\right)\left|\rho_{e q}\right\rangle=\left(\begin{array}{c}
\rho_{z}^{e q} \\
0 \\
0
\end{array}\right)\left|\rho_{+}^{\lambda}\right\rangle=\left(\begin{array}{c}
0 \\
\lambda^{\prime} \\
i \lambda^{\prime}
\end{array}\right)\left|\rho_{o}\right\rangle=\left(\begin{array}{c}
\rho_{z} \\
0 \\
0
\end{array}\right)
$$

The first vector is a general density vector, the second one corresponds to the equilibrium value, the third one corresponds to the detection vector, and the last one to a density vector with only the z component not zero. The scalar product of a density vector with $\left|\rho_{+}^{\lambda}\right\rangle$ gives the detected complex signal

$$
s\left(t, \lambda^{\prime}\right)=\left\langle\rho_{+}^{\lambda} \mid \rho^{\lambda}(t)\right\rangle=\lambda^{\prime}\left(\rho_{x}^{\lambda}(t)+i \rho_{y}^{\lambda}(t)\right)
$$


$\langle A \mid B\rangle$ is the scalar product between vectors $|A\rangle$ and $|B\rangle$.

Explicit matrices of the super-operators corresponding to free evolution and pulse periods, that apply for one orientation of the crystallite with respect to the coil axis, that is for a given value of $\lambda^{\prime}$, are presented below. The superscript ${ }^{\lambda}$ is no longer written for clarity. Definitions and abbreviations are given in Table.1.

\begin{tabular}{l|l}
\hline$\beta=\omega_{R F} t_{p}$ & $v=\omega_{N} \beta$ \\
$\Delta x=\frac{\Delta \omega}{\omega_{R F}}$ & $e_{q t}=\exp \left(-t / T_{1 q}\right)$ \\
$\omega_{N}=\sqrt{\Delta x^{2}+\lambda^{\prime 2}}$ & $e_{2 t}=\exp \left(-t / T_{2}\right)$ \\
$c_{u}=\cos (u)=\frac{\lambda^{\prime}}{\omega_{N}}$ & $c_{v}=\cos (v)$ \\
$s_{u}=\sin (u)=\frac{\Delta x}{\omega_{N}}$ & $s_{v}=\sin (v)$ \\
\hline
\end{tabular}

Table 1: Definitions and abbreviations used to write the super-operators for NQR of spins $\mathrm{I}=3 / 2$. At resonance, $\Delta x=0, \omega_{N}=\lambda^{\prime}, c_{u}=1, s_{u}=0, v=\lambda^{\prime} \beta$.

The free propagator $\mathbb{E}_{t}$ is

$$
\mathbb{E}_{t}=\left(\begin{array}{ccc}
e_{q t} & 0 & 0 \\
0 & e_{2 t} * \cos (\Delta \omega t) & -e_{2 t} * \sin (\Delta \omega t) \\
0 & e_{2 t} * \sin (\Delta \omega t) & e_{2 t} * \cos (\Delta \omega t)
\end{array}\right)
$$

The pulse super-operators are

$$
\mathbb{P}_{x}=\left(\begin{array}{ccc}
c_{u}^{2}\left(c_{v}-1\right)+1 & c_{u} * s_{u}\left(1-c_{v}\right) & c_{u} * s_{v} \\
c_{u} * s_{u}\left(1-c_{v}\right) & c_{v}+c_{u}^{2}\left(1-c_{v}\right) & -s_{u} * s_{v} \\
-c_{u} * s_{v} & s_{u} * s_{v} & c_{v}
\end{array}\right)
$$


and

$$
\mathbb{P}_{y}=\left(\begin{array}{ccc}
c_{u}^{2}\left(c_{v}-1\right)+1 & -c_{u} * s_{v} & c_{u} * s_{u}\left(1-c_{v}\right) \\
c_{u} * s_{v} & c_{v} & -s_{u} * s_{v} \\
c_{u} * s_{u}\left(1-c_{v}\right) & s_{u} * s_{v} & c_{v}+c_{u}^{2}\left(1-c_{v}\right)
\end{array}\right)
$$

As an example, the signal recorded after a $\mathbb{P}_{x}$ pulse when the system is initially with a density vector that has only its $\mathrm{z}$ component not equal to zero $\left|\rho_{o}\right\rangle^{T} \sim\left(\rho_{z}, 0,0\right)$, like for instance the equilibrium density vector, is calculated as follows :

$$
\begin{aligned}
s_{P}\left(t, \lambda^{\prime}\right) & =\left\langle\rho_{+}^{\lambda}\left|\mathbb{E}_{t} \mathbb{P}_{x}\right| \rho_{o}\right\rangle+\left\langle\rho_{+}^{\lambda}\left|\left(\mathbb{I}-\mathbb{E}_{t}\right)\right| \rho_{o}\right\rangle \\
& =\left\langle\rho_{+}^{\lambda}\left|\mathbb{E}_{t} \mathbb{P}_{x}\right| \rho_{o}\right\rangle \\
& =\left(\begin{array}{ll}
0 \lambda^{\prime} i \lambda^{\prime}
\end{array}\right) \mathbb{E}_{t} \mathbb{P}_{x}\left(\begin{array}{c}
\rho_{z} \\
0 \\
0
\end{array}\right) \\
& =\rho_{z}\left[\frac{\lambda^{\prime 2} \Delta x}{\omega_{N}^{2}}\left(1-\cos \left(\omega_{N} \beta\right)\right)-i \frac{\lambda^{\prime 2}}{\omega_{N}} \sin \left(\omega_{N} \beta\right)\right] e^{i \Delta \omega t} e^{-t / T_{2 q}}
\end{aligned}
$$

From these super-operators, the steady-state density vector and acquisition signal can be readily calculated from Eq.6, 7 and 17. The solutions are valid for a single crystal experiment.

For a one pulse sequence, The steady-state signal recorded just after the last pulse of the sequence is

$$
\begin{aligned}
s_{s s t P}\left(t, \lambda^{\prime}\right) & =\left\langle\rho_{+}^{\lambda}\left|\mathbb{E}_{t} \mathbb{P}\right| \rho_{s s t}\right\rangle+\left\langle\rho_{+}^{\lambda}\left|\left(\mathbb{I}-\mathbb{E}_{t}\right)\right| \rho_{e q}\right\rangle \\
& =\left\langle\rho_{+}^{\lambda}\left|\mathbb{E}_{t} \mathbb{P}\right| \rho_{s s t}\right\rangle
\end{aligned}
$$


The steady-state signal of a two-pulse sequence is

$$
\begin{aligned}
s_{s s t P P}\left(t, \lambda^{\prime}\right) & =\left\langle\rho_{+}^{\lambda}\left|\mathbb{E}_{t}\left(\mathbb{P}_{2} \mathbb{E}_{\tau_{2}} \mathbb{P}_{1}\right)\right| \rho_{s s t}\right\rangle+\left\langle\rho_{+}^{\lambda}\left|\left(\mathbb{I}-\mathbb{E}_{t}+\mathbb{E}_{t} \mathbb{P}_{2}\left(\mathbb{I}-\mathbb{E}_{\tau_{2}}\right)\right)\right| \rho_{e q}\right\rangle \\
& =\left\langle\rho_{+}^{\lambda}\left|\mathbb{E}_{t}\left(\mathbb{P}_{2} \mathbb{E}_{\tau_{2}} \mathbb{P}_{1}\right)\right| \rho_{s s t}\right\rangle+\left\langle\rho_{+}^{\lambda}\left|\left(\mathbb{E}_{t} \mathbb{P}_{2}\left(\mathbb{I}-\mathbb{E}_{\tau_{2}}\right)\right)\right| \rho_{e q}\right\rangle
\end{aligned}
$$

The last contribution is the free induction decay due to the relaxation between the two pulses, and excited by the second pulse of the sequence. This contribution is proportional to $1-\exp \left(-\tau_{2} / T_{1 q}\right) \approx \tau_{2} / T_{1 q}$ because $\tau_{2} / T_{1 q} \ll 1$. For a real echo sequence where $\tau_{2} \sim T_{2 q} \ll T_{1 q}$, this contribution is very small and can be neglected $s_{P P}\left(t, \lambda^{\prime}\right) \approx\left\langle\rho_{+}^{\lambda}\left|\mathbb{E}_{t}\left(\mathbb{P}_{2} \mathbb{E}_{\tau_{2}} \mathbb{P}_{1}\right)\right| \rho_{\text {sst }}\right\rangle$.

When a powder sample is used, a steady-state signal is calculated for each value of $\lambda^{\prime}$, the signal from Eq.22, 23 and the powder average is calculated from Eq.15. The results and comparison with experiments are described in the next section.

\section{Results}

In all this section, the case of a recycle time $T$ that is large enough to let the coherences decay to zero $\left(T_{2 q} \ll T\right)$ is considered. In practice, it corresponds to most experimental conditions. But a word of caution should again be added with regards to the hypothesis of neglecting coherence refocussing. If this is not verified, then the FID could be distorted in phase and intensity, and the theory presented in this section is no longer valid. How coherences spoil the signal in repetitive experiments is well documented for spins $I=1 / 2$ (see for instance [26]). From the theoretical point of view, all calculation are greatly simplified when $T_{2 q} \ll T$, because the free evolution propagator $\mathbb{E}_{T}$ reduces to a very simple matrix which is zero everywhere except the compo- 
nent $\mathbb{E}_{T z z}=e_{q T}$. The main results are compared to ${ }^{35} \mathrm{Cl}$ NQR experiments performed on chloranil.

\subsection{Simulations, Sample and experimental methods}

The numerical simulation were directly performed in the Liouville space, or with explicit formula, using home-made functions implemented in Matlab [27]. Matlab was installed on a laptop Dell Precision M6600 with 8 GB of memory, and running an Intel(R) Core(TM) i7-2820QM CPU 2.3GHz. The most efficient implementation of powder averaging other all directions is to generate a set of values of $\lambda^{\prime}$ from an isotropic distribution of $(\theta, \phi)$ angles $\left(10^{6}\right.$ or $\left.1.610^{7}\right)$, to calculate the signal $S\left(t, \lambda^{\prime}\right)$ for each $\lambda^{\prime}$, and to take the mean value at the end.

The experiments were performed on chloranil powder $\left(\mathrm{C}_{6} \mathrm{Cl}_{4} \mathrm{O}_{2}\right.$ tetrachloro1,4-benzoquinone) with a Bruker AVANCE I 300 spectrometer, using a static Bruker probe.

At ambient temperature, the ${ }^{35} \mathrm{Cl}$ NQR of chloranil gives two lines around 36.8MHz and 36.87MHz Fig.2(Top), with asymmetry parameter of both lines $\eta \approx 0.2([28][29][30])$. We only used the low frequency line LF. The RF power was $\nu_{R F}=8$ or $9.5 \mathrm{kHz}$.

The spin-lattice relaxation time of the LF line was measured with an inversion-recovery sequence. The best least-square fit with the usual three parameter fit function $S(\tau)=a-b e^{-c \tau}$ where $c=1 / T_{1 q}$ leads to $T_{1 q} \approx 15 \mathrm{~ms}$ (see Fig.2(Bottom)). The coherence relaxation time $T_{2 q}$ was measured by varying the delay between the two pulses of a solid-echo sequence, giving $T_{2 q} \approx 2.7 \mathrm{~ms}$.

Since the theory applies only when the coherences have decayed, the 
minimum recycle time should not be smaller that typically $5 \times T_{2 q} \approx 13.5 \mathrm{~ms}$, a value close to $T_{1 q}$. It limits the minimum recycle time to $T_{1 q}$. However, for experimental reasons due to our spectrometer electronics, the minimum recycle time that could be reached was $T>25 \mathrm{~ms}$. All experiments were performed for $T / T_{1 q} \geq 2$.

The NQR spectra were acquired after a number of dummy scans of 64 that ensures that the steady-state is reached. For variable pulse length experiments, the maximum pulse length (or pulse angle) was limited by probe arcing or electrical breakdown. Single pulse and two-pulse solid-state echo were used.

\subsection{Steady-state of a one-pulse sequence}

Within the hypothesis of a complete decay of the coherences during the recycle time, the steady-state vector is only along $z$, and was directly calculated from Eq.6

$$
\rho_{s s t, z}=\frac{1-\exp \left(-T / T_{1 q}\right)}{1-\exp \left(-T / T_{1 q}\right) \mathbb{P}_{z z}} \rho_{e q, z}
$$

with $\mathbb{P}_{z z}$ the $z z$ component of the pulse super-operator. This formula is valid on or out of resonance, and is completely similar to the spin $I=1 / 2$ case and $\mathrm{I}=1$ case [22], as expected from the formal analogies described in the previous sections. The consequences of this formula are discussed for NQR, mainly for powder samples.

\subsubsection{On resonance : optimum signal and $S N R$}

On resonance, Eq.24 leads to :

$$
\rho_{s s t, z}=\frac{1-\exp \left(-T / T_{1 q}\right)}{1-\exp \left(-T / T_{1 q}\right) \cos \left(\lambda^{\prime} \beta\right)} \rho_{e q, z}
$$



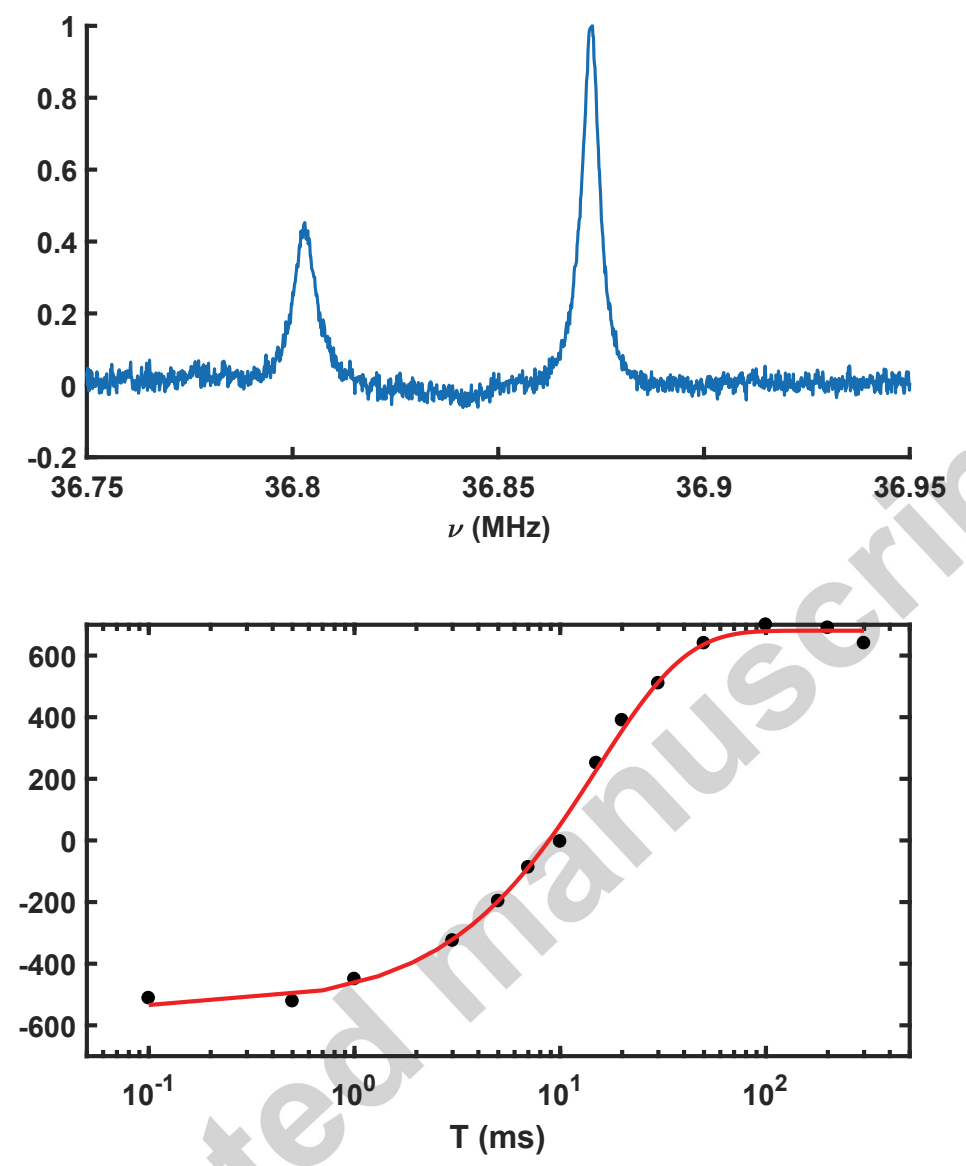

Figure 2: (Top) Typical ${ }^{35} \mathrm{Cl}$ NQR chloranil spectrum at ambient temperature ; (Bottom) Inversion-recovery experiment : LF line integral as a function of waiting time. The continuous line is the best least-square fit that gives a spin-lattice relaxation time $T_{1 q} \approx 15$ ms.

The corresponding signal is

$$
S(t)=\langle S(t, \lambda)\rangle=\left\langle\lambda^{\prime} \frac{1-\exp \left(-T / T_{1 q}\right)}{1-\exp \left(-T / T_{1 q}\right) \cos \left(\lambda^{\prime} \beta\right)} \sin \left(\lambda^{\prime} \beta\right)\right\rangle
$$

where $\left\langle\right.$. $>$ denotes an average over all orientations or $\lambda^{\prime}$ values present in the sample. For a single crystal, only one $\lambda^{\prime}$ value is needed. If $\lambda^{\prime} \equiv 1$, we recover the NMR case Eq.2 as expected. 
For a single crystal, the angle $\beta_{M}^{\lambda}$ that gives the maximum signal follows $\cos \left(\lambda^{\prime} \beta_{M}^{\lambda}\right)=\exp \left(-T / T_{1 q}\right)$, a relationship similar to the Ernst equation Eq.3.

Typical nutation curves for a single crystal are presented in Fig.3(Top). For each $\eta$, three values of $\lambda^{\prime}$ were considered, the minimum and maximum values, as well as the median. The maximum and nutation curve shape strongly depends on the orientation of the coil in the EFG PAS, and $T / T_{1 q}$.

The question is now to determine the optimum angle for an isotropic powder, as a function of $\eta$. Simulated nutation curves for a powder sample are presented in Fig.3(Bottom) for $\eta=0,0.5,1$. For a given $\eta$, both the angle giving the maximum signal $\beta_{M}(\eta)$ and the maximum values of the curve are decreasing functions of $T / T_{1 q}$. As can be seen, the effect of the asymmetry parameter on the nutation curves is very small, and can be hardly distinguished by eye. It is a remarkable consequence of the powder average, and of some peculiar properties of the directional parameter $\lambda^{\prime}$ whose mean and dispersion are almost $\eta$ independent.

The powder averaged signal can be analytically differentiated as a function of pulse angle $\beta$, and the zero of this function will give the maximum signal angle. The result is a non-linear equation that can be solved numerically for each $\eta$ and ratio $T / T_{1 q}$ :

$$
\left\langle\left(\lambda^{\prime}\right)^{2} \frac{\cos \left(\lambda^{\prime} \beta\right)-\exp \left(-T / T_{1 q}\right)}{\left[1-\exp \left(-T / T_{1 q}\right) \cos \left(\lambda^{\prime} \beta\right)\right]^{2}}\right\rangle=0
$$

For completeness, we also studied the pulse angle $\beta_{0}$ that gives a zero signal. In NMR with hard pulses, it would correspond to a $\pi$ or $180^{\circ}$ pulse. The results for both angles are presented in Fig.4(Top).

Both the optimum angle $\beta_{M}(\eta)$ and $\beta_{o}$ only slightly depend on $\eta$. $\beta_{M}$ is an 

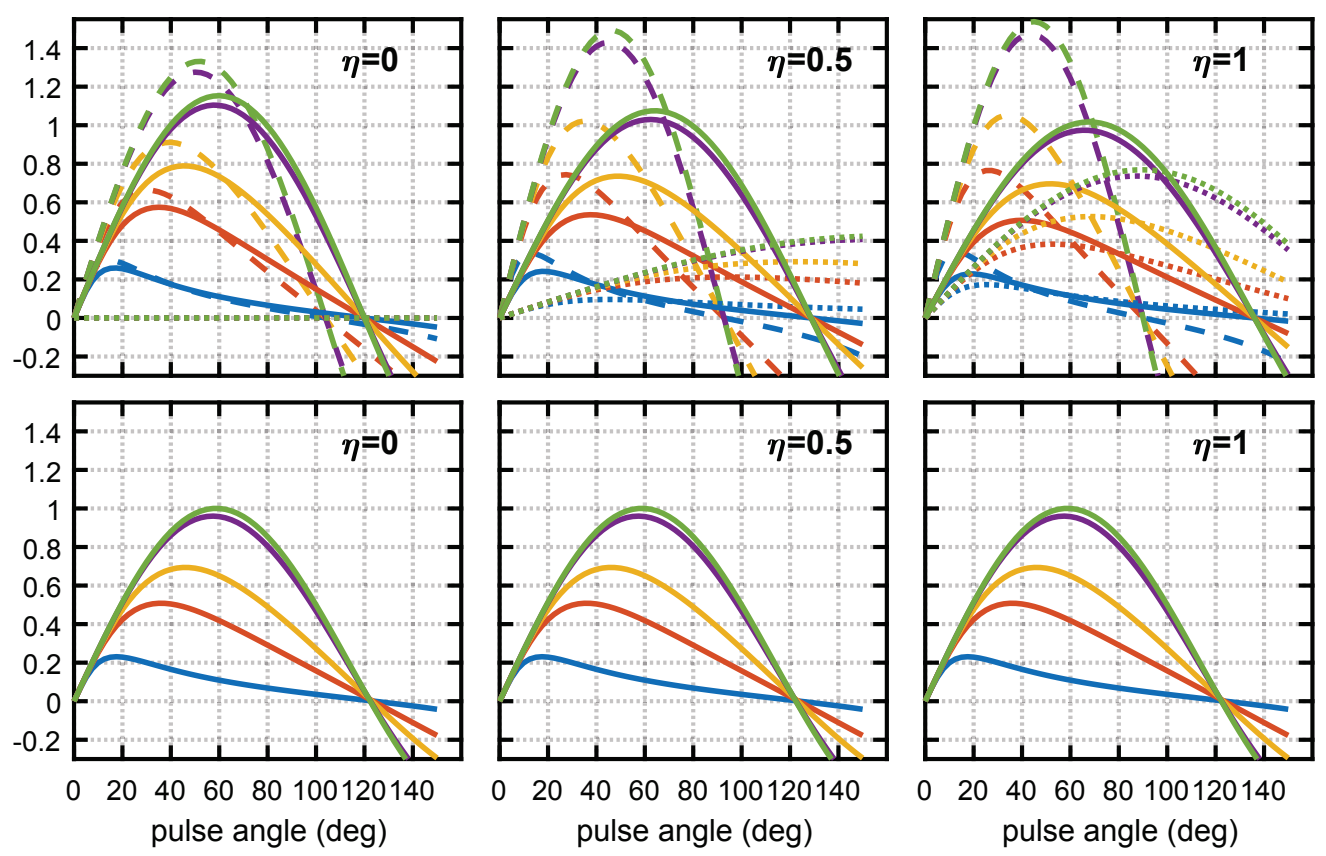

Figure 3: NQR nutation curves for a single pulse sequence at resonance for $\eta=0,0.5$ and 1. (Top) Single crystal nutation curves for three values of $\lambda^{\prime}$ : (dashed line) Maximum of $\lambda^{\prime}$, (solid line) Median of $\lambda^{\prime}(1.5,1.4,1.32$ for $\eta=0,0.5$ and 1 respectively), (dotted line) Minimum of $\lambda^{\prime}$. (Bottom) Powder averaged nutation curves. For each $\eta$, the ratio $T / T_{1 q}$ is from top to bottom $5,3,1,0.5,0.1$. All the nutation curves were normalized such that the powder nutation curve at $\eta=0$ and $T / T_{1 q}=5$ has a maximum equal to one.

increasing function of $T / T_{1 q}$, that reaches almost $60^{\circ}$ for a completely relaxed system. The angle is smaller than in NMR $\left(90^{\circ}\right)$ because of the directional parameter $\lambda^{\prime}>1$. $\beta_{o}$ is almost constant, it varies within $[120,124.5]^{\circ}=[2.09,2.17]$ rad whatever $\eta$ and $T / T_{1 q}$.

In analogy with NMR, we looked for a relationship between the cosinus of this angle as a function of $E=\exp \left(-T / T_{1 q}\right)$. A good fit could be obtained 

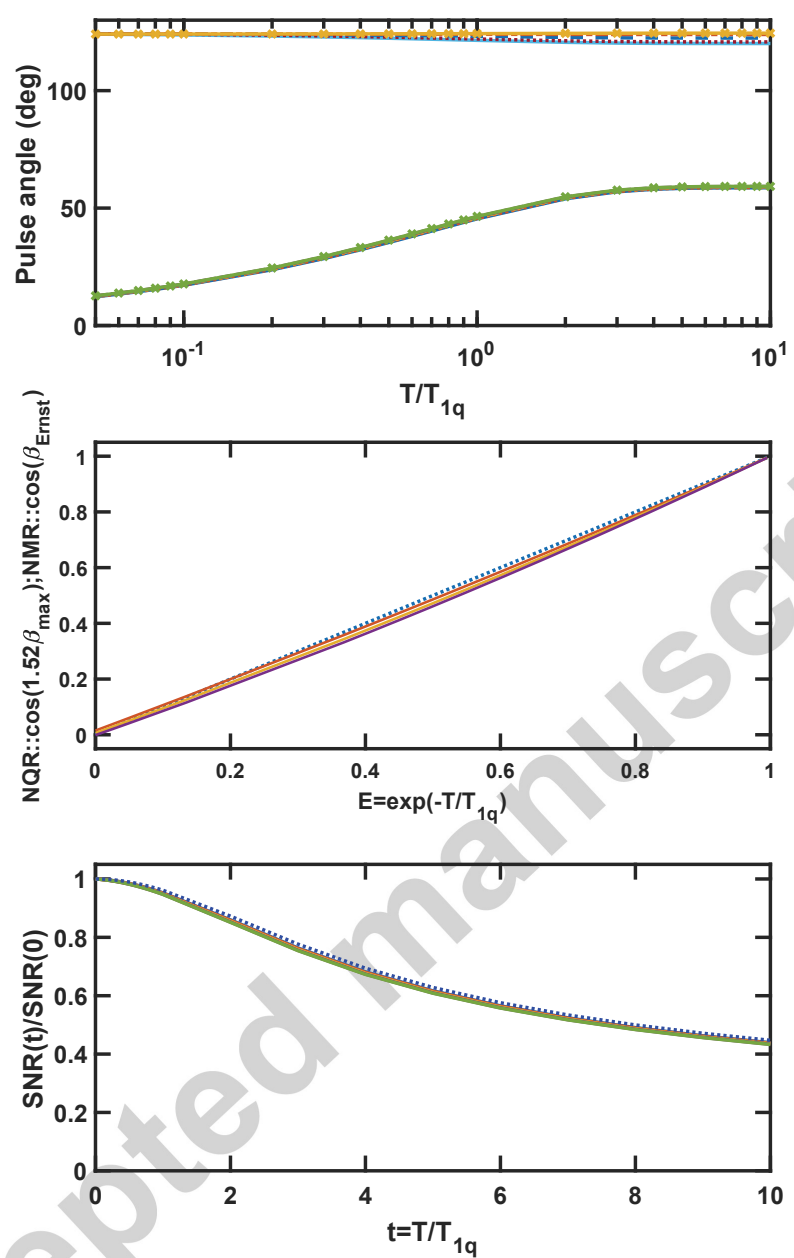

Figure 4: (Top) Angle $\beta_{M}$ that gives the maximum signal $\left(\beta_{M} \leq 60^{\circ}\right)$, and $\beta_{0}$ for the first zero of the nutation curve (from top to bottom, $\eta=0$ to 1 ). Note that the x-axis is in logarithmic scale. ;(Middle) dotted line : $\cos \left(\beta_{\text {Ernst }}\right)=E$ for NMR where $E=$ $\exp \left(-T / T_{1}\right):$ this is the bisector. Colored continuous lines $\cos \left(1.52 \beta_{M}\right)$ vs $E$ where $E=\exp \left(-T / T_{1 q}\right)$ for each $\eta$ (from top to bottom, $\eta=0$ to 1 ). ; (Bottom) Normalized $\operatorname{SNR}(\mathrm{T}) / \operatorname{SRN}(0)$ as a function of $T / T_{1 q}$. The NMR optimal SNR is given by a dotted line. 
as $\cos \left(\beta_{M}\right)=a+b E$ for each $\eta$.

However, we also found that $\cos \left(f \beta_{M}\right) \approx E$ whatever $\eta$ to a good approximation when $f=1.52$. These curves are plotted on Fig.4(Middle). First, the curves only slightly differ when $\eta$ varies, and the relationship to $E$ is almost linear. Although it is not perfectly linear, they are all very close to the bisector corresponding to the NMR Ernst formula, with square correlation factor larger than 0.98 as indicated by the last line of Table.2. This table also summarized the result of a linear fit of $\cos (1.52 \beta)$ as a function of $E$, and the slopes are very close to one, with very small intercepts and large square correlation factor $R^{2}>0.998$.

\begin{tabular}{l|c|c|c|c|c}
\hline & $\eta=0$ & $\eta=0.25$ & $\eta=0.5$ & $\eta=0.75$ & $\eta=1$ \\
\hline slope & 0.98211 & 0.98449 & 0.99014 & 0.99524 & 0.99708 \\
intercept & 0.0084287 & 0.0048277 & -0.0034242 & -0.010632 & -0.013159 \\
$R^{2}$ & 0.99957 & 0.99944 & 0.99912 & 0.9988 & 0.99868 \\
\hline$R^{2}$ with bisector & 0.98313 & 0.98373 & 0.98496 & 0.98591 & 0.98622 \\
\hline
\end{tabular}

Table 2: Results of a linear fit of $\cos (1.52 \beta)$ as a function of $E=\exp \left(-T / T_{1 q}\right)$. The last line gives the correlation coefficients of the NQR results with respect to the bisector.

Thus, for a powder sample, the angle giving the maximum NQR signal for a one pulse steady-state experiment can be cast in a form analogous to the Ernst Formula :

$$
\cos \left(1.52 \beta_{M}\right) \approx \exp \left(-T / T_{1 q}\right)
$$

Following the analogy, we looked whether an NMR-like formula would 


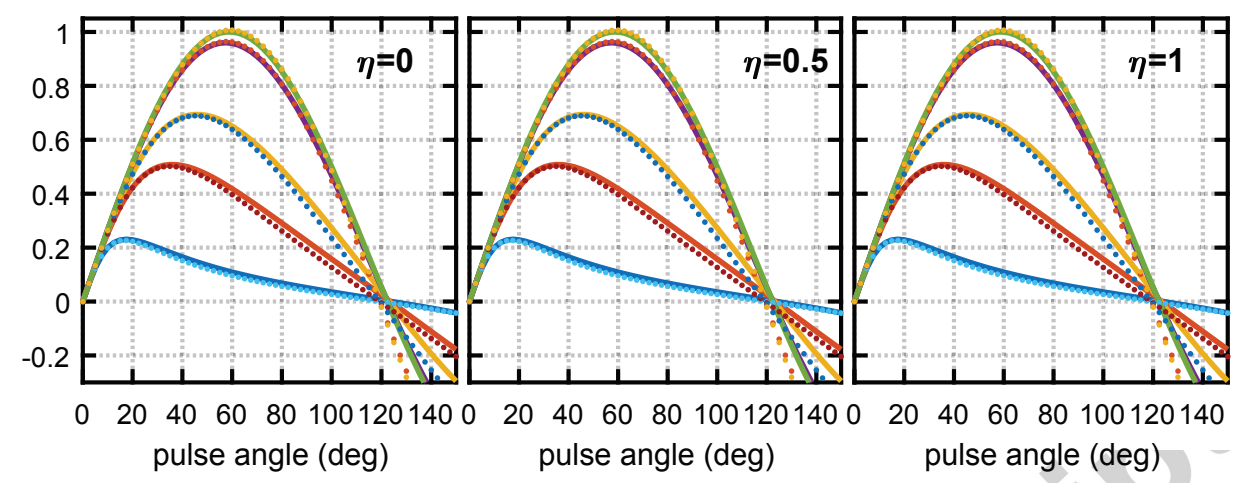

Figure 5: NQR powder averaged nutation curves for a single pulse sequence (solid lines). For each $\eta$, the ratio $T / T_{1 q}$ is from top to bottom $5,3,1,0.5,0.1$. Dots are the corresponding nutation curves from the simplified formula Eq.29.

represent the NQR powder averaged nutation curves

$$
S_{a}(t)=\frac{1-\exp \left(-T / T_{1 q}\right)}{1-\exp \left(-T / T_{1 q}\right) \cos (1.52 \beta)} \sin (1.52 \beta)
$$

Figure.5 presents the superposition of real NQR nutation curves and simplifed formula as a function of nutation angle for three values of $\eta$. The nutation curves are well approximated by Eq.29 for angles less than the optimal angle Eq.28, and the discrepancy increases for larger pulse angles. However, the overall behavior is well reproduced, and this formula can be used as a rule of thumb to estimate the expected NQR nutation curve from a powder sample.

In practical cases, the question is to obtain the maximum SNR for a given acquisition time, given a pulse angle that gives the maximum signal. The signal $s\left(T / T_{1 q}\right)$ is a function of $t_{q}=T / T_{1 q}$, an a-dimensional variable. Given a number $N$ of signal accumulations, the SNR will be $S N R \propto \sqrt{N} s\left(t_{q}\right)$. Using $N \sim 1 / T$ with $\mathrm{T}$ the recycle time, leads to 


$$
S N R\left(t_{q}\right) \sim \frac{s\left(t_{q}\right)}{\sqrt{t_{q}}}
$$

In NMR, Using the signal given by Eq.2, and the Ernst pulse angle, the normalized SNR is

$$
\operatorname{snr}\left(t_{q}\right)=\frac{S N R\left(t_{q}\right)}{S N R(0)}=\sqrt{\frac{2}{t_{q}} \frac{\left(1-e^{-t_{q}}\right)}{\left(1+e^{-t_{q}}\right)}}
$$

where here, for NMR, $t_{q}=T / T_{1}$ with $T_{1}$ the spin-lattice (longitudinal) relaxation time. The SNR was normalized to 1 for $t \mapsto 0$. snr $\left(t_{q}\right)$ is a decreasing function of $t_{q}$.

We numerically calculated $\frac{S N R\left(t_{q}\right)}{S N R(0)}$ for NQR, by evaluating Eq.26 at $\beta=$ $\beta_{M}(\eta)$, and the SNR from Eq.30.

After normalization, the results are reported on Fig.4(bottom) for each $\eta$, as well as the NMR result. The NQR curves are practically independent of the asymmetry parameter, and almost overlap the NMR result. As in NMR, reducing the recycle time increases the SNR if the pulse angle is chosen to give the maximum signal. But the gain is quite small, snr $\approx 0.63$ when $T=5 T_{1 q}$ and $s n r \approx 0.97$ when $T=T_{1 q}$.

3.3. One pulse at resonance and out of resonance : comparison with experiments

The steady state density vector for a single pulse out of resonance was found as

$$
\rho_{s s t, z}=\frac{1-\exp \left(-T / T_{1 q}\right)}{1-\exp \left(-T / T_{1 q}\right)\left[1+\left[\left(\frac{\lambda^{\prime}}{\omega_{N}}\right)^{2}\left(\cos \left(\lambda^{\prime} \beta\right)-1\right)\right]\right]} \rho_{e q, z}
$$


It is easy to verify that this expression reduces to Eq. 25 when $\Delta x=0$ because $\omega_{N}=\lambda^{\prime}$. The signal was then calculated from Eq.21, and averaged over all $\lambda^{\prime}$ values.

Figure.6 compares the theoretical calcultations to experimental points obtained from ${ }^{35} \mathrm{Cl} \mathrm{NQR}$ on chloranil powder. No free parameters are used for the theoretical calculation $\left(T_{1 q}, T_{2 q}, \omega_{R F}\right.$ and $\Delta \omega$ are known), except that the signal amplitude was normalized to one when $T / T_{1 q}=5$ for both theory and experiment. As shown by Fig. 6 the agreement between theory and experiment is good for on or out of resonance excitation.

\subsection{Two pulse echo sequence at resonance}

To avoid dead time problems, a solid-echo sequence $\beta_{x}-\tau-\beta_{y}$ sequence is often used to acquire NQR spectra. From Eq.7, the steady state z-component $\rho_{z}$ reduces to

$$
\rho_{\infty, z}=\frac{1-\exp \left(-T / T_{1 q}\right)\left[1-\left(1-\exp \left(-\tau / T_{1 q}\right)\right) \cos \left(\lambda^{\prime} \beta\right)\right]}{1-\exp \left(-T / T_{1 q}\right) \exp \left(-\tau / T_{1 q}\right) \cos ^{2}\left(\lambda^{\prime} \beta\right)} \rho_{e q, z}
$$

Again, it is valid for a given orientation of the coil in the EFG PAS. This expression is slightly more complicated than for the single pulse sequence because the interpulse delay $\tau$ contributes to population relaxation, and the two pulses give a contribution to the denominator. For an echo sequence, $\tau \ll T, \tau$ being chosen large enough to avoid the dead-time, but as small as possible with respect to $T_{2 q}$ to reduce the signal loss due to the $e^{-2 \tau / T_{2 q}}$ factor.

That Eq.33 formula is consistent with the single pulse sequence steadystate can be seen by putting $\tau=T$. In that case, the repetitive solid-echo 

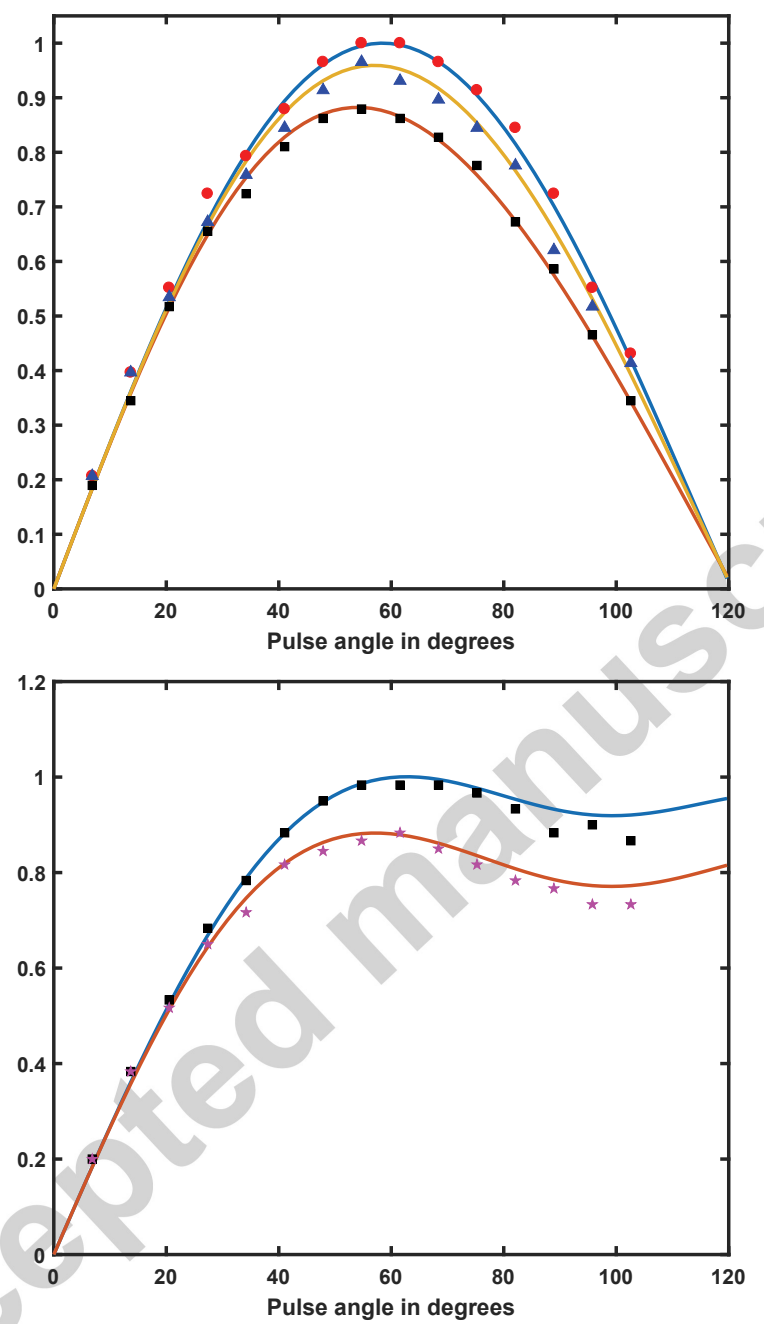

Figure 6: Steady state signal for a one pulse sequence as a function of ratio $T / T_{1 q}$ and pulse angle for the LF line of chloranil. $\omega_{R F} /(2 \pi)=9.5 \mathrm{kHz}$. Points are experimental values while continuous lines are theoretical results with no free parameters. (Top) At resonance. From top to bottom, $T / T_{1 q}=5,3,2$; (Bottom) Out of resonance with $\Delta \omega=10 \mathrm{kHz}$ that is $\Delta x=\Delta \omega / \omega_{R F}=1.053$. From top to bottom, $T / T_{1 q}=5,2$. 
pulse sequence reduces to the repetition of a single pulse, calculated over two periods instead of one. We thus expect to obtain the same formula as Eq.25. When $\tau=T$, Eq.33 reduces to (assuming $\rho_{e q, z} \equiv 1$ for simplicity)

$$
\begin{aligned}
\rho_{\infty, z} & =\frac{1-e_{q T}\left[1-\left(1-e_{q T}\right) \cos \left(\lambda^{\prime} \beta\right)\right]}{1-e_{q T}^{2} \cos ^{2}\left(\lambda^{\prime} \beta\right)}=\frac{\left(1-e_{q T}\right)\left(1+e_{q T} \cos \left(\lambda^{\prime} \beta\right)\right)}{1-\left(e_{q T} \cos \left(\lambda^{\prime} \beta\right)\right)^{2}} \\
& =\frac{\left(1-e_{q T}\right)\left(1+e_{q T} \cos \left(\lambda^{\prime} \beta\right)\right)}{\left(1-e_{q T} \cos \left(\lambda^{\prime} \beta\right)\right)\left(1+e_{q T} \cos \left(\lambda^{\prime} \beta\right)\right)}=\frac{\left(1-e_{q T}\right)}{\left(1-e_{q T} \cos \left(\lambda^{\prime} \beta\right)\right)}
\end{aligned}
$$

This is exactly the formula obtained for a single pulse sequence.

The echo amplitude, or spectrum, is proportional to

$$
\begin{gathered}
S_{P P}(t)=\langle S(t, \lambda)\rangle \\
=\left(1-e_{q T}\right)\left\langle\frac{\left[1-\left(1-e_{q \tau}\right) \cos \left(\lambda^{\prime} \beta\right)\right]}{1-e_{q T} e_{q \tau} \cos ^{2}\left(\lambda^{\prime} \beta\right)} \lambda^{\prime} \sin \left(\lambda^{\prime} \beta\right)\left(1-\cos \left(\lambda^{\prime} \beta\right)\right)\right\rangle
\end{gathered}
$$

A comparison of Eq.34 with experiments is presented in Fig.7. Again, the agreement of theory and experiments is good.

\section{Conclusion}

Explicit formula for the steady-state signal generated by one and twopulse sequences repetitive experiments were obtained and analysed for NQR of spins $\mathrm{I}=3 / 2$, using a Liouville space approach combined with a specific formalism for NQR of spins $I=3 / 2$. The main hypothesis to obtain explicit simple formula is the decay to zero of the coherences during the recycle time.

The effect of powder averaging that affects the excitation and the detection in NQR is discussed in detail. For a single pulse experiment, ErnstAnderson-like formula for the optimum pulse angle and SNR were obtained 


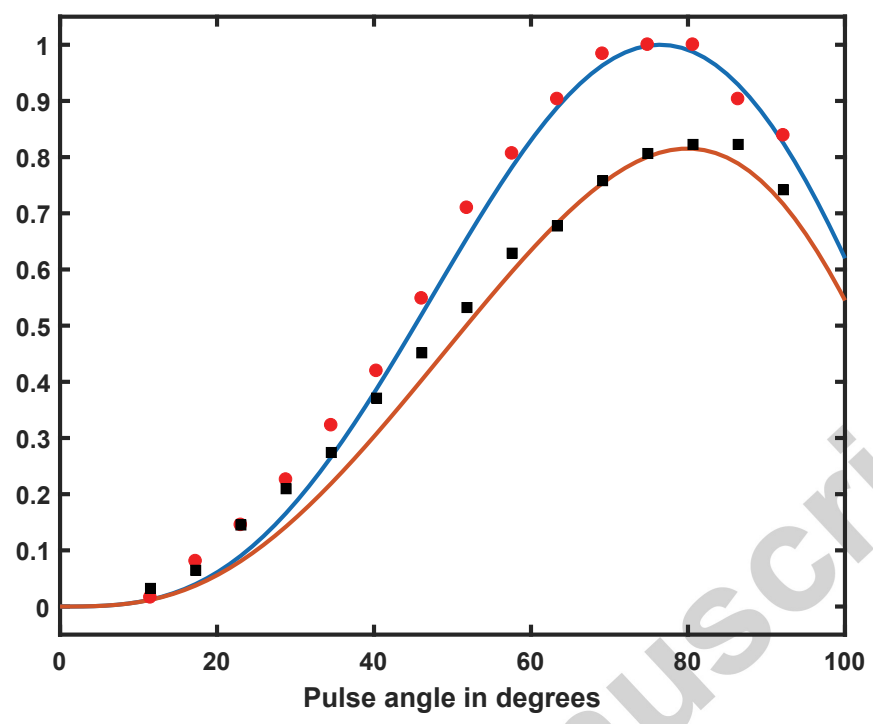

Figure 7: Steady state signal for a two pulse solid echo $\left(\beta_{x}\right)-\tau-\left(\beta_{y}\right)$ sequence at resonance as a function of ratio $T / T_{1 q}$ and pulse angle for the LF line of chloranil. Points are experimental values while continuous lines are theoretical results with no free parameters. $\omega_{R F} /(2 \pi)=9.5 \mathrm{kHz}, \tau=50 \mu \mathrm{s}$. From top to bottom, $T / T_{1 q}=5,2$.

for single crystal and powder samples, and compared to the corresponding results in NMR of spins $\mathrm{I}=1 / 2$. The solid-echo steady-state signal was also obtained. These theoretical results are compared and validated by ${ }^{35} \mathrm{Cl} \mathrm{NQR}$ experiments with a chloranil powder sample.

All these results show that the Liouville-space formalism is an essential tool in deriving NMR/NQR results. From the practical point of view, this article show that recycle times as short as $T \approx T_{1 q}$ can be used to acquire and accumulate NQR of spins $\mathrm{I}=3 / 2$ signals.

Further work are in progress to apply this approach to quadrupolar NMR of spins $\mathrm{I}=3 / 2$. 
Funding : This research received FEDER financial support (FEDER 34722Prin2Tan) for funding NMR spectrometers.

Acknowledgments : I would like to thank Doctor Bertrand Toudic for his support, and Claire Roiland and Laurent Le Polles for fruitfull discussions.

[1] B. H. Suits, Nuclear Quadrupole Resonance Spectroscopy, in "'Handbook of Applied Solid State Spectroscopy"' edited by Vij, D. R., Springer US Boston, MA, 65-96 (2006).

[2] G. P. Wulfsberg, Nuclear Quadrupole Resonance (NQR) Spectroscopy, in "'Encyclopedia of Inorganic and Bioinorganic Chemistry"', John Wiley \& Sons, Ltd (2011).

[3] H. G. Dehmelt, H. Kruger, Kernquadrupolfrequenzen in festem Dichlorathylen, Naturwissenschaften 37 111-112 (1950).

[4] H. G. Dehmelt, H. Kruger, Kernquadrupolfrequenzen in kristallinen Jodverbindungen, Naturwissenschaften 37 398-398 (1950).

[5] H. G. Dehmelt, Quadrupolresonanzfrequenzen des kristallinen Broms, Z. Physik 130 480-482 (1951).

[6] H. G. Dehmelt, H. Kruger, Quadrupol-Resonanzfrequenzen von Cl- und Br-Kernen in kristallinem Dichlorthylen und Methylbromid, Z. Physik 129 401-415 (1951).

[7] M. Bloom, E. L. Hahn, B. Herzog, Free magnetic induction in nuclear quadrupole resonance Phys. Rev. 97, 1699-1709 (1955). 
[8] T. P. Das, E.L.Hahn, Nuclear Quadrupole Resonance Spectroscopy, Academic Press, New York,(1958).

[9] E. Balchin, D. J. Malcolme-Lawes, I. J. F. Poplett, M. D. Rowe, J. A. S. Smith, G. E. S. Pearce, and, S. A. C. Wren, Potential of Nuclear Quadrupole Resonance in Pharmaceutical Analysis, Analytical Chemistry 77 (13), 3925-3930 (2005).

[10] J. N. Latosinnska, Nuclear Quadrupole Resonance spectroscopy in studies of biologically active molecular systemsa review, Journal of Pharmaceutical and Biomedical Analysis 38 , 577-587 (2005).

[11] S. C. Perez and L. Cerioni and A. E. Wolfenson and S. Faudone and S. L. Cuffini, Utilization of pure nuclear quadrupole resonance spectroscopy for the study of pharmaceutical crystal forms, International Journal of Pharmaceutics 298, 143-152 (2005).

[12] J. N. Latosinnska, Applications of nuclear quadrupole resonance spectroscopy in drug development, Expert Opinion on Drug Discovery $\mathbf{2}$, $225-248$ (2007).

[13] S. Limandri, C. Visnovezky, S. C. Perez, C. A. Schurrer, A. E. Wolfenson, M. Ferro, S. L. Cuffini, J. Gonalves de Souza, F. A. Aguiar, and C. M. de Gaitani, Nuclear Quadrupole Resonance: A Technique to Control Hydration Processes in the Pharmaceutical Industry, Analytical Chemistry 83 (5), 1773-1776 (2011).

[14] C. Chen, F. Zhang,J. Barras, K. Althoefer, S. Bhunia and S. Mandal, Authentication of Medicines Using Nuclear Quadrupole Resonance 
Spectroscopy, IEEE/ACM Transactions on Computational Biology and Bioinformatics 13, 417-430 (2016).

[15] H.Y. Carr, Steady-State Free Precession in Nuclear Magnetic Resonance, Phys. Rev. 112, 1693-1701 (1958).

[16] R.R. Ernst and W.A. Anderson, Application of Fourier transform spectroscopy to magnetic resonance, Rev. Sci. Instrum. 37, 93-102 (1966).

[17] R. Freeman and H.D.W. Hill, Fourier transform study of NMR spin lattice relaxation by "progressive saturation", J. Chem. Phys. 54,33673377 (1971)

[18] R. Freeman and H.D.W. Hill, Phase and intensity anomalies in Fourier transform NMR, J. Magn. Reson. 4, 366-383 (1971).

[19] D.E. Jones and H. Sternlicht, Fourier transform nuclear magnetic resonance I.repetitive pulses, J. Magn. Reson. 6, 167-182 (1972).

[20] M. Ferrari, N. Hiblot, A. Retournard, D. Canet, 14N pulsed nuclear quadrupole resonance. 3. Effect of a pulse train. Optimal conditions for data averaging, Molecular Physics 105, 3005-3012 (2007).

[21] http://www.pascal-man.com/periodic-table/periodictable.html

[22] Christophe Odin, Quadrupolar solid-state $\{\mathrm{NMR}\}$ and repetitive experiments: Some aspects in the Liouville space. Application to spins I=1, Solid State Nuclear Magnetic Resonance in press, corrected proof Available online 29 December 2016, http://dx.doi.org/10.1016/j.ssnmr.2016.12.012. 
[23] Christophe Odin, Calculations of Multipulse Sequence in NQR of Spins 3/2, J. Magn. Reson. 141, 239-255 (1999).

[24] Christophe Odin, Broadband Echo Sequence Using a $\pi$ Composite Pulse for the Pure NQR of a Spin I = 3/2 Powder Sample, J. Magn. Reson. $143,299-310$ (2000).

[25] J. C. Pratt, P. Raghunathan, and C. A. McDowell, Transient response of a quadrupolar spin system in zero applied field, J. Magn. Reson. 20, 313327 (1975).

[26] R.R. Ernst, G. Bodenhausen, and A. Wokaun, "Principles of Nuclear Magnetic Resonance in One and Two Dimensions", Clarenton Press, Oxford, 124-132 (1994).

[27] MATLAB and Symbolic Math Toolbox(Version 7.0) R2016a, The MathWorks, Inc., Natick, Massachusetts, United States.

[28] S. S. C. Chu, G. A. Jeffrey and T. Sakurai, The crystal structure of tetrachloro-p-benzoquinone (chloranil), Acta Cryst. 15, 661-671 (1962).

[29] C. B. Richardson, Temperature Dependence of the Zeeman Effect in the Nuclear Quadrupole Resonance in Chloranil, J. Chem. Phys. 38:2, 510-515 (1963).

[30] G. S. Harbison, A. Slokenbergs, and T. M. Barbara, Two-dimensional zero-field nutation nuclear quadrupole resonance spectroscopy, J. Chem. Phys. 90, 5292-5298 (1989). 